\title{
Effects of Exercise on Gene Expression of Inflammatory Markers in Human Peripheral Blood Cells: A Systematic Review
}

\author{
Gyrd O. Gjevestad $^{1,2}$ • Kirsten B. Holven ${ }^{1,3}$ - Stine M. Ulven ${ }^{4}$
}

Published online: 21 May 2015

(C) The Author(s) 2015. This article is published with open access at Springerlink.com

\begin{abstract}
Regular physical activity seems to be one of the most important contributors to prevent disease and promote health. Being physically active reduces the risk of developing chronic diseases such as cardiovascular disease, diabetes, and some types of cancers. The molecular mechanisms are however not fully elucidated. Depending on duration and intensity, exercise will cause disruption of muscle fibers triggering a temporary inflammatory response. This response may not only involve the muscle tissue, but also peripheral tissues such as white blood cells, which are important components of the immune system. The immune system plays a vital role in the development of atherosclerosis, thereby making white blood cells relevant to study when looking at molecular mechanisms induced by physical activity. In this review, we summarize the existing literature on exercise and gene expression in human white blood cells, and discuss these results in relation to inflammation and atherosclerosis.
\end{abstract}

Keywords Physical activity · Exercise · Gene expression . Inflammation - Atherosclerosis · Peripheral mononuclear blood cells · PBMCs $\cdot$ Leukocytes · Lymphocytes .

Monocytes

This article is part of the Topical Collection on Physical Activity

Stine M. Ulven

StineMarie.Ulven@hioa.no

1 University of Oslo, Oslo, Norway

2 TINE SA, Oslo, Norway

3 Norwegian National Advisory Unit on Familial Hypercholesterolemia, Oslo University Hospital, Oslo, Norway

4 Oslo and Akershus University College of Applied Sciences, Oslo, Norway

\section{Introduction}

There are substantial epidemiological evidence that regular physical activity (exercise) reduce the risk of developing diseases such as cardiovascular disease [1-4], type 2 diabetes [2, $4,5]$, and some types of cancers $[2,4,6]$. Regular physical activity is therefore one of the most important contributors to maintaining health. The mechanisms by which exercise contributes to health are however not fully understood.

An acute bout of exercise, depending on type, intensity, and duration [7-9], causes tissue injury, triggering a local and systemic inflammation with a release of both pro- and anti-inflammatory cytokines $[10,11]$, while regularly physical activity seems to attenuate the inflammatory response promoting an anti-inflammatory environment in the body $[3,12,13]$.

The inflammatory response may be studied through changes in circulating biomarkers, such as interleukins, chemokines, and other signaling molecules. When studying possible effects of exercise in relation to atherosclerosis, biomarkers for underlying processes, such as endothelial dysfunction, oxidative stress, and inflammation are relevant (Table 1).

Peripheral white blood cells are important components of the immune system, and the immune system is important in the development of atherosclerosis [14]. White blood cells are constantly interacting with other cells, such as endothelial cells in the arteries [15-17], making them relevant for studying the inflammatory process in atherosclerosis [17-19].

It is vital for the body to regulate the expression of genes in the process of adapting to changes in the environment, such as exercise [20]. Gene expression studies can be used to get an insight into molecular mechanisms in affected cells, and to reflect the early stages of activation in the immune system. Gene expression studies may therefore be a sensitive tool to characterize the early effects of exercise on immune regulation [12]. 
Table 1 Common inflammatory markers, included in this review, and their biological functions relevant for atherosclerosis and physical activity

\begin{tabular}{|c|c|c|}
\hline Inflammatory markers & $\begin{array}{l}\text { Gene } \\
\text { symbol }\end{array}$ & Function \\
\hline Chemokine (C-C motif) ligand 2 & CCL2 & Involved in chemotactic activity for monocytes and basophils, binding to CCR 2 and CCR4. \\
\hline Chemokine (C-C motif) ligand 3 & CCL3 & Involved in the acute inflammation by recruitment and activation of leukocytes. \\
\hline Chemokine (C-C motif) ligand 4 & CCL4 & Involved in the migration of immune cells, a chemoattractant. \\
\hline Chemokine (C-C motif) ligand 5 & CCL5 & Involved in recruiting leukocytes to inflammatory sites. \\
\hline $\begin{array}{l}\text { Chemokine (C-C motif) } \\
\text { receptors }(2,3 \text { and } 4)\end{array}$ & $\mathrm{CCR} 2,3,4$ & Involved in the regulation of cell trafficking, important in inflammation, binds to cytokines. \\
\hline $\begin{array}{l}\text { Chemokine (C-X-C motif) } \\
\text { ligand } 16\end{array}$ & CXCL16 & Involved in the migration of immune cells, a chemoattractant. \\
\hline Endothelial nitric oxide synthase & NOS3 & Involved in the generation of $\mathrm{NO}$ in blood vessels, regulating vascular tone, and platelet aggregation. \\
\hline GATA binding protein 3 & GATA3 & T cell-specific transcription factor involved in the regulation of $\mathrm{T}$ cell development. \\
\hline Glutathione peroxidase & GPX & Involved in the detoxification of hydrogen peroxide. \\
\hline $\begin{array}{l}\text { Heat shock } 27 \text { kDa protein- } \\
\text { associated protein } 1\end{array}$ & HSPBAP1 & Involved in stress resistance; actin organization, and translocation from the cytoplasm to the nucleus. \\
\hline Heat shock $70 \mathrm{kDa}$ protein $1 \mathrm{~A}$ & HSPA1A & $\begin{array}{l}\text { Involved in stress resistance; stabilizing proteins against aggregation and mediates the folding of newly } \\
\text { translated proteins. }\end{array}$ \\
\hline Heat shock $70 \mathrm{kDa}$ protein 6 & HSPA6 & Involved in stress resistance; protein folding, stabilization, and shuttling functions in response to stress. \\
\hline Inducible nitric oxide synthase & NOS2 & Involved in immune response and important in cellular signaling, produces NO. \\
\hline Interferon gamma & IFNG & Involved in the regulation of immune and inflammatory response, promotes Th1 differentiation. \\
\hline Interleukin 1 receptor antagonist & IL1RN & $\begin{array}{l}\text { Inhibits the activity of IL1A/IL1B, and modulates a variety of interleukin 1-related immune and } \\
\text { inflammatory responses. }\end{array}$ \\
\hline Interleukin 1 receptor-like 1 & IL1R1 & Involved in cytokine-induced immune and inflammatory response. \\
\hline Interleukin 10 & IL10 & $\begin{array}{l}\text { Downregulates the expression of Th1 cytokines, enhances B cell survival, proliferation, and antibody } \\
\text { production, able to block NF- } \mathrm{kB} \text { activity. }\end{array}$ \\
\hline Interleukin 13 & IL13 & $\begin{array}{l}\text { Immunoregulatory cytokine that plays a role in B cell maturation and differentiation, downregulates } \\
\text { macrophage activity inhibiting the production of pro-inflammatory cytokines and chemokines. }\end{array}$ \\
\hline Interleukin $1 \mathrm{a}$, interleukin $1 \mathrm{~b}$ & IL1A/IL1B & Proliferation and maturation of lymphocytes, involved in inflammation and acute-phase response. \\
\hline Interleukin 4 & IL4 & $\begin{array}{l}\text { Pleiotropic cytokine involved in } \mathrm{T} \text { cell and macrophage differentiation and modulate the differentiation } \\
\text { to Th2. }\end{array}$ \\
\hline Interleukin 6 & IL6 & A pleiotropic cytokine that plays important roles in inflammation and the acute-phase response. \\
\hline Interleukin 8 & IL8 & Involved in the acute inflammatory response, a chemoattractant. \\
\hline Matrix metallopeptidase 9 & MMP9 & Involved in the breakdown of extracellular matrix and tissue remodeling. \\
\hline NADPH oxidase & $\begin{array}{l}\text { NADPH } \\
\text { oxidase }\end{array}$ & Involved in the vascular superoxide production. \\
\hline $\begin{array}{l}\text { Prostaglandin-endoperoxide } \\
\quad \text { synthase } 2\end{array}$ & PTGS2 & An enzyme responsible for formation of prostanoids, involved in inflammation. \\
\hline Superoxide dismutase 1 & SOD1 & Involved in the anti-oxidative defense destroying free superoxide radicals in the body. \\
\hline Superoxide dismutase 2 & SOD2 & Involved in the anti-oxidative defense destroying free superoxide radicals in the body. \\
\hline TNF receptor-associated factor 6 & TRAF6 & Involved in signal transducing in NF-kappa B pathway. \\
\hline Toll-like receptors 2,4 , and 7 & $\begin{array}{l}\text { TLR2, } 4 \text {, } \\
\text { and } 7\end{array}$ & $\begin{array}{l}\text { Involved in recognition of pathogen-associated molecular patterns (PAMPs), mediate the production of } \\
\text { cytokines necessary for the development of effective immunity. }\end{array}$ \\
\hline Transforming growth factor beta & TGFB & Involved in proliferation, differentiation, adhesion, and migration. \\
\hline Tumor necrosis factor alpha & TNF & $\begin{array}{l}\text { Prototypical pro-inflammatory cytokine, plays a central role in inflammation, immune system devel- } \\
\text { opment, and apoptosis. }\end{array}$ \\
\hline
\end{tabular}

We have summarized gene expression studies in human white blood cells, including peripheral mononuclear blood cells (PBMCs), lymphocytes and monocytes, but excluding natural killer (NK) cells. Studies, both acute exercise interventions and interventions studies investigating the prolonged effects of exercise, have been included.

\section{Literature Search}

A literature search was conducted in a combined search in Ovid Medline and EMBASE in November 2014 and re-ran in February 2015. Medical subject headings (MeSH) combined with words, some of them truncated, in title or abstract (tw) or only in title (ti), was used as follows: leukocytes, 
mononuclear/ or exp lymphocyte subsets/ or exp b-lymphocytes/ or exp t-lymphocytes/ or monocytes/ or (leukocyte* or mononuclear or monocytes or lymphocyte* or macrophage*).tw. AND exp exercise/ or exp physical endurance/ or (exercise* or physical activit* or athlete*).tw. or training.ti. or Athletes/ AND gene expression/ or exp transcription, genetic/ or gene expression regulation/ or downregulation/ or gene amplification/ or protein modification, translational/ or protein processing, post-translational/ or rna processing, post-transcriptional/ or transcriptional activation/ or up- regulation/ OR (gene expression or transcriptom or rna or mrna).tw. exp RNA AND exp Cytokines/ or exp Inflammation/ or exp Immune System Phenomena/ or (cytokine* or interleukin* or inflammat* or immun*).tw.

The search was limited to humans with: not animals (including studies were data on humans and animals were reported separately) and to articles published in English, Danish, Norwegian, or Swedish.
After removing duplicates, 565 papers were identified. Only original papers and papers including invention studies with leukocytes, including PBMCs, monocytes, lymphocytes, and dendrite cells, were included. Studies were excluded if only whole blood was analyzed, no gene expression data were presented, or the intervention included giving food supplements in combination with exercise. Studies including NK cells only were not included. Neither were papers including subjects on a weight reduction program. Using these criteria, the number of relevant articles was 78. Another 46 papers were excluded after reading the abstract/full article. Additional two articles, identified from the reference lists of the already included papers, were found to be relevant and included in the review. After a re-run of the search, three new articles were included in the review. In total, 37 papers are included in the review as shown in Fig. 1.
Fig. 1 Flow chart showing the result of the combined literature search in Ovid Medline and EMBASE and the selection of papers

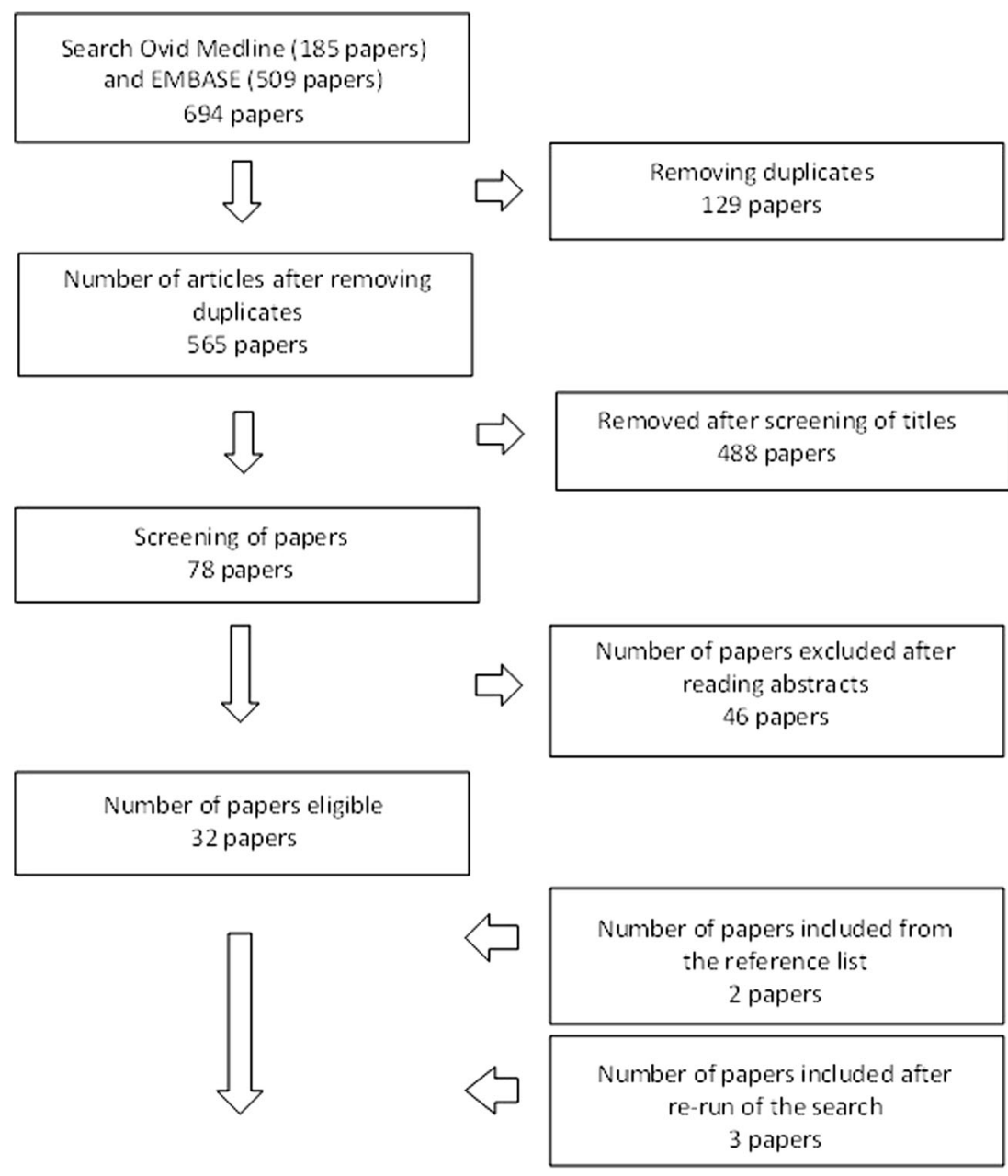

Number of papers included 37 papers 


\section{Acute Exercise and Gene Expression Studies}

Table 2a summarizes the effect of an acute bout of exercise on gene expression in white blood cells from 28 studies. In 26 of the studies, the subjects performed an acute bout of endurance exercise, while in the remaining two studies subjects performed an acute bout of strength exercise. In 18 of the studies, PBMCs were investigated, while in the other studies leukocytes (seven studies), lymphocytes (two studies), and monocytes (one study) were investigated.

\section{Gene Expression Studies in PBMCs-Endurance Training}

Peripheral blood mononuclear cells (PBMCs) are a subpopulation of leukocytes and consist of approximately $70 \% \mathrm{~T}$ lymphocytes, 5-10 \% B lymphocyte, $15 \%$ monocytes, 10 $15 \%$ natural killer (NK) cells, and $0.5-1 \%$ dendritic cells [54] and are often used to study the immune response in relation to atherosclerosis [18].

Ullum et al. were one of the first to publish a paper where gene expression in PBMCs in response to exercise was studied [21]. They measured gene expression of interleukin (IL) 1A, IL1B, IL6, and tumor necrosis factor alpha (TNF) before and after an ergometric bicycle exercise, but did not find any effect on gene expression after exercise. These results were largely supported by Moldoveanu et al. [25], Bernecker et al. [35], and Natelson et al. [22]. Ostrowski et al. concluded slightly differently when showing that PBMC gene expression of interleukin 1 receptor antagonist (IL1RN) and IL1B were upregulated after a marathon race [23]. Xiang et al. showed an upregulation of messenger ribonucleic acid (mRNA) levels 1 week after a marathon race for several genes, among them IL4, GATA binding protein 3 (GATA3), chemokine (C-C-motif) receptor (CCR)4, CCR3, and CCR2 [37]. A change in the Th1/Th2 ratio was observed from pre- to post-marathon as interferon gamma (IFNG)/IL4 ratio and T cell-specific T-box transcription factor T-bet (TBX21)/GATA3 ratio decreased. In agreement with Xiang el al. [37], Ulven et al. showed an upregulation of GATA3 mRNA expression in PBMCs after ergometric cycling [36]. mRNA expression of IL1B, chemokine (C-X-C motif) ligand 16 (CXCL16), IL8, prostaglandinendoperoxide synthase 2 (PTGS2), and TBX21 were upregulated, while toll-like receptor 2 (TLR2) mRNA expression was downregulated after exercise. Li et al. found an increase in the mRNA gene expression of the anti-inflammatory cytokine IL13 after one hour tai chi [32].

Toll-like receptors (TLRs) play an important role in the immune system by recognizing and initiating an inflammatory response to dangerous molecules, possibly leading to the transcription of cytokines and chemokines [55, 56]. Nickel et al. investigated how a marathon race affected the expression of
TLRs in lean subjects exercising regularly compared to lean subjects and obese subjects exercising less regularly [33]. They found differences in mRNA expression of TLR4 and TLR7 between the groups.

There is some evidence that a reduced level of nitric oxide (NO) or an elevated level of superoxide $\left(\mathrm{O}_{2}^{-}\right)$increase the risk of cardiovascular disease. The expression and activity of inducible nitric oxide synthase (NOS2) relative to endothelial nitric oxide synthase (NOS3) is important in the regulation of inflammation [31]. Jenkins et al. compared the mRNA expression of several genes related to the antioxidant defense system in sedentary and physically active males after a treadmill test [31]. In CD34 PBMCs, NOS3 gene expression increased in the sedentary group compared to the active group after exercise. No change was seen in the expression level of NOS2 between groups. Niess et al. found an increase in the expression of NOS2 after a marathon race, but not after a graded treadmill test [26].

The abovementioned studies were performed using reverse transcription polymerase chain reaction (RT-PCR). When using RT-PCR, only a limited number of genes can be analyzed at the same time. With a whole genome transcriptomic approach, it is possible to analyze thousands of genes at the same time.

When using this approach, Kimsa et al. found that an acute bout of bicycling regulated several biological pathways such as cytokine-mediated signaling pathways (IL6, IL8, and IL1B), intracellular signaling (IL5RA, IL6, and IL8), cell communication and cell-to-cell signaling (interleukin 2 receptor beta (IL2RB), colony-stimulating factor 2 (CSF2), and interleukin 1 receptor-like 1 (IL1R1). In total, ten inflammation-related genes were changed after exercise [44].

Connolly et al. showed that 311 genes were altered in PBMCs from baseline to immediately after a cycle ergometer workout, 552 genes were regulated from end of exercise and 60 min into recovery while 292 genes were regulated between baseline and $60 \mathrm{~min}$ into recovery [38]. The majority of the genes upregulated from baseline to end of exercise were related to inflammation and stress. From end of exercise and $60 \mathrm{~min}$ into recovery, an upregulation of nuclear receptor subfamily 4, group A, member 2 (NR4A2) and regulator of $\mathrm{G}$ protein signaling 1 (RGS1) were observed. The mRNA levels of IL6 and IL10 were not affected by the exercise at any time point.

Radom-Aizik et al. investigated how a bout of ergometric cycling altered gene expression in PBMCs in early and late puberty females [13] and males [12]. They observed that there were differences in gene expression between genders and pubertal phase. Genes commonly regulated by exercise in all groups were related to growth, apoptosis, inflammation, and tissue repair.

Transforming growth factor beta (TGFB) is involved in proliferation and differentiation. Kimsa et al. identified 14 


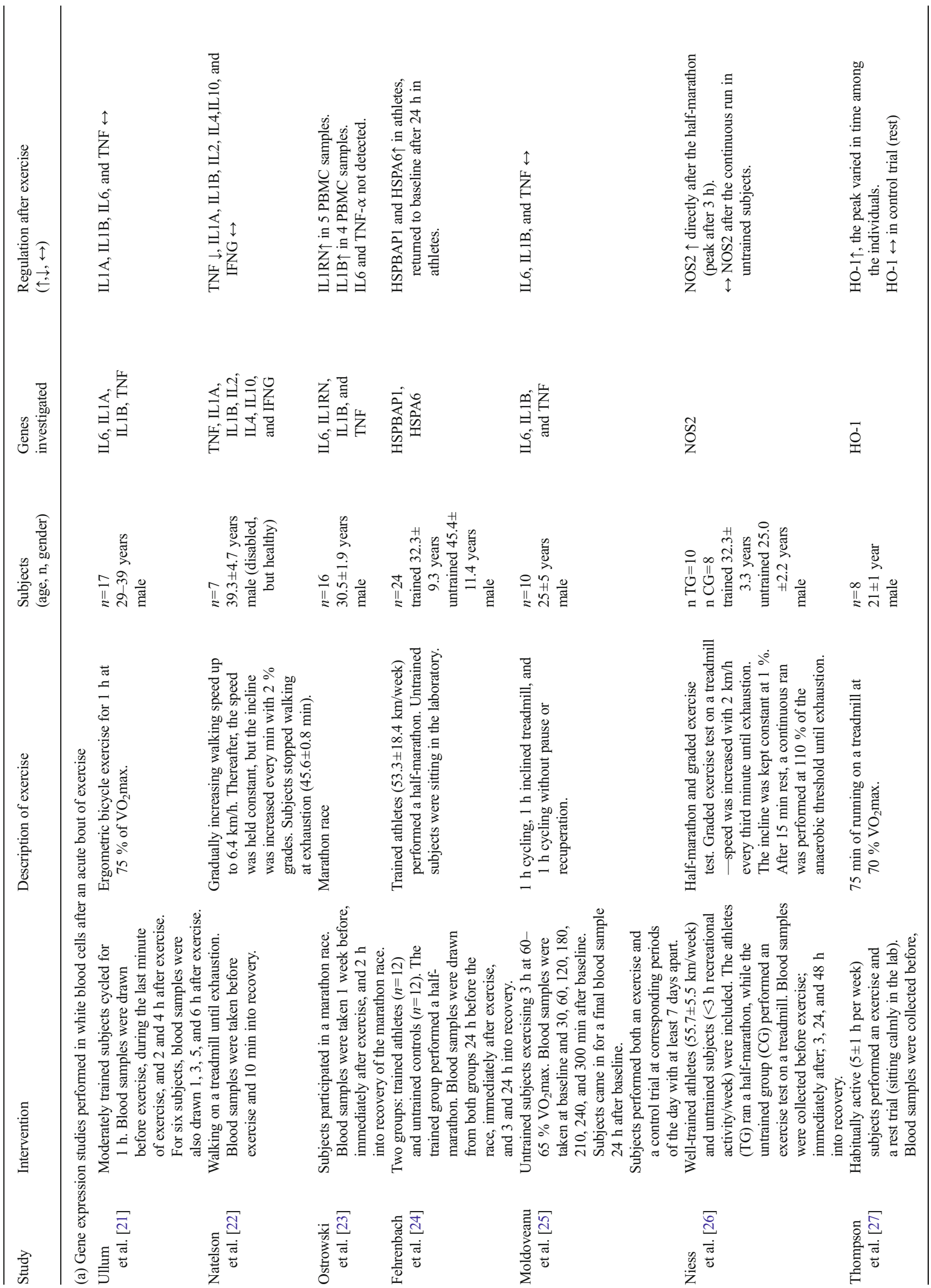




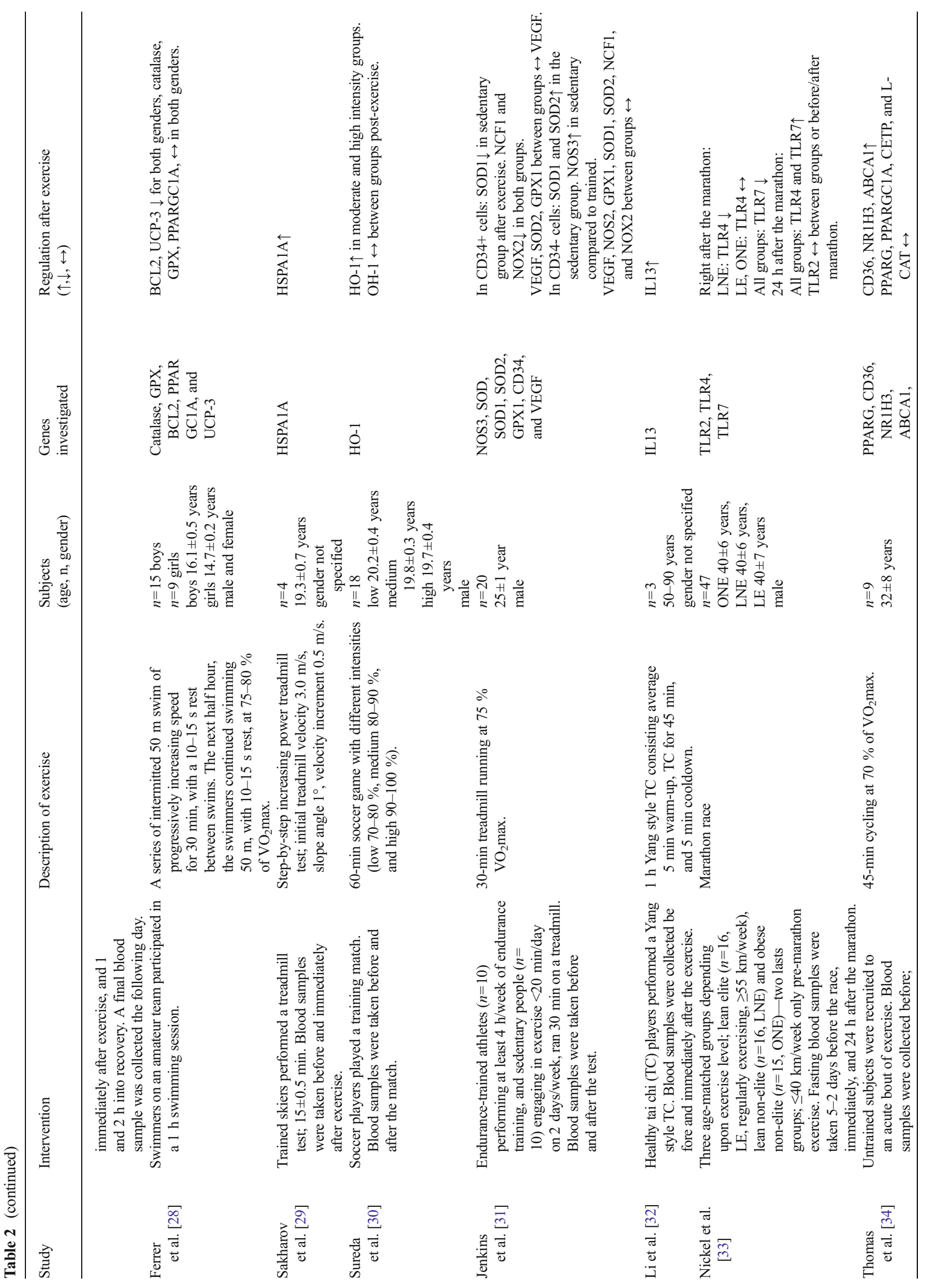




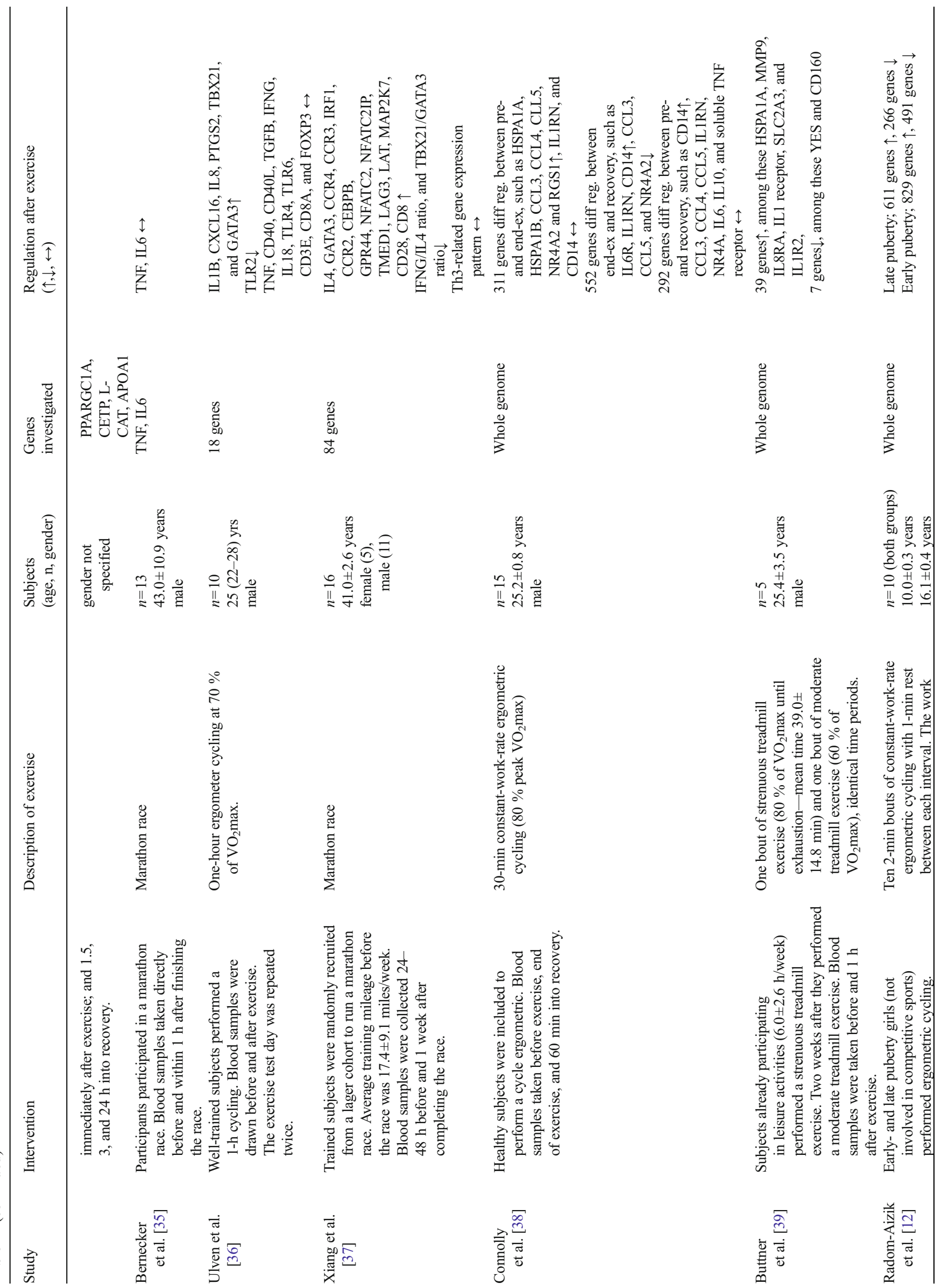




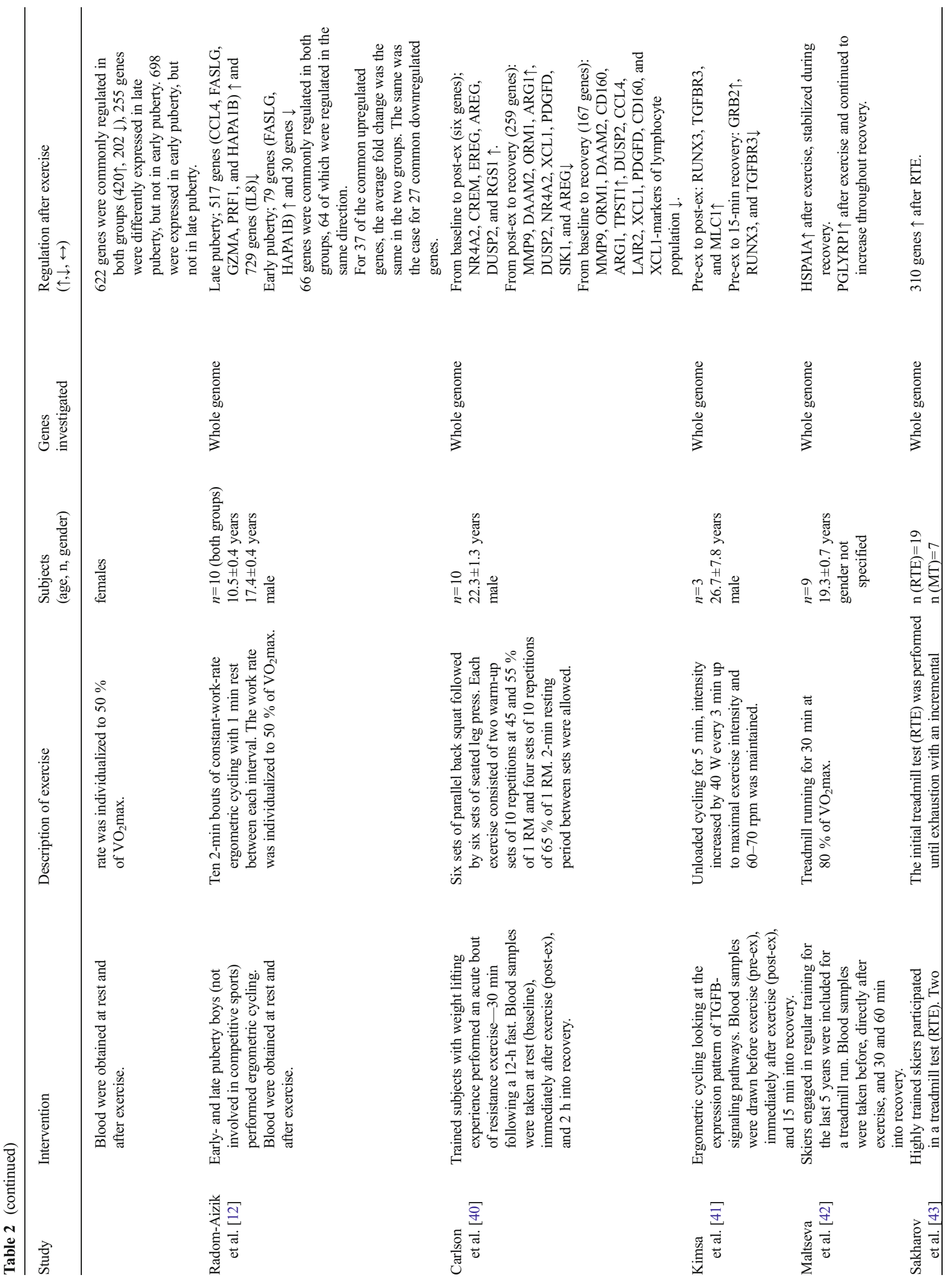




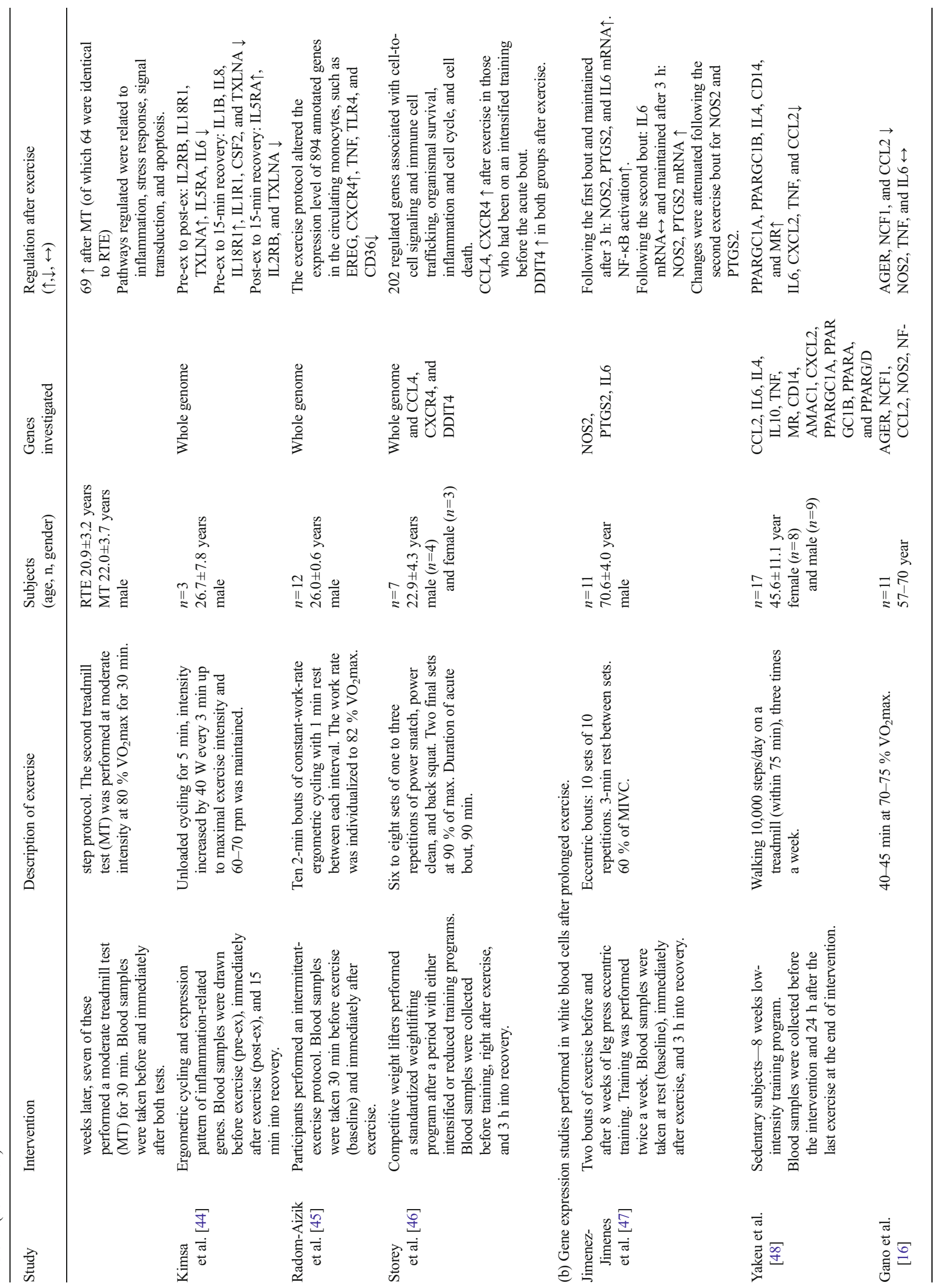




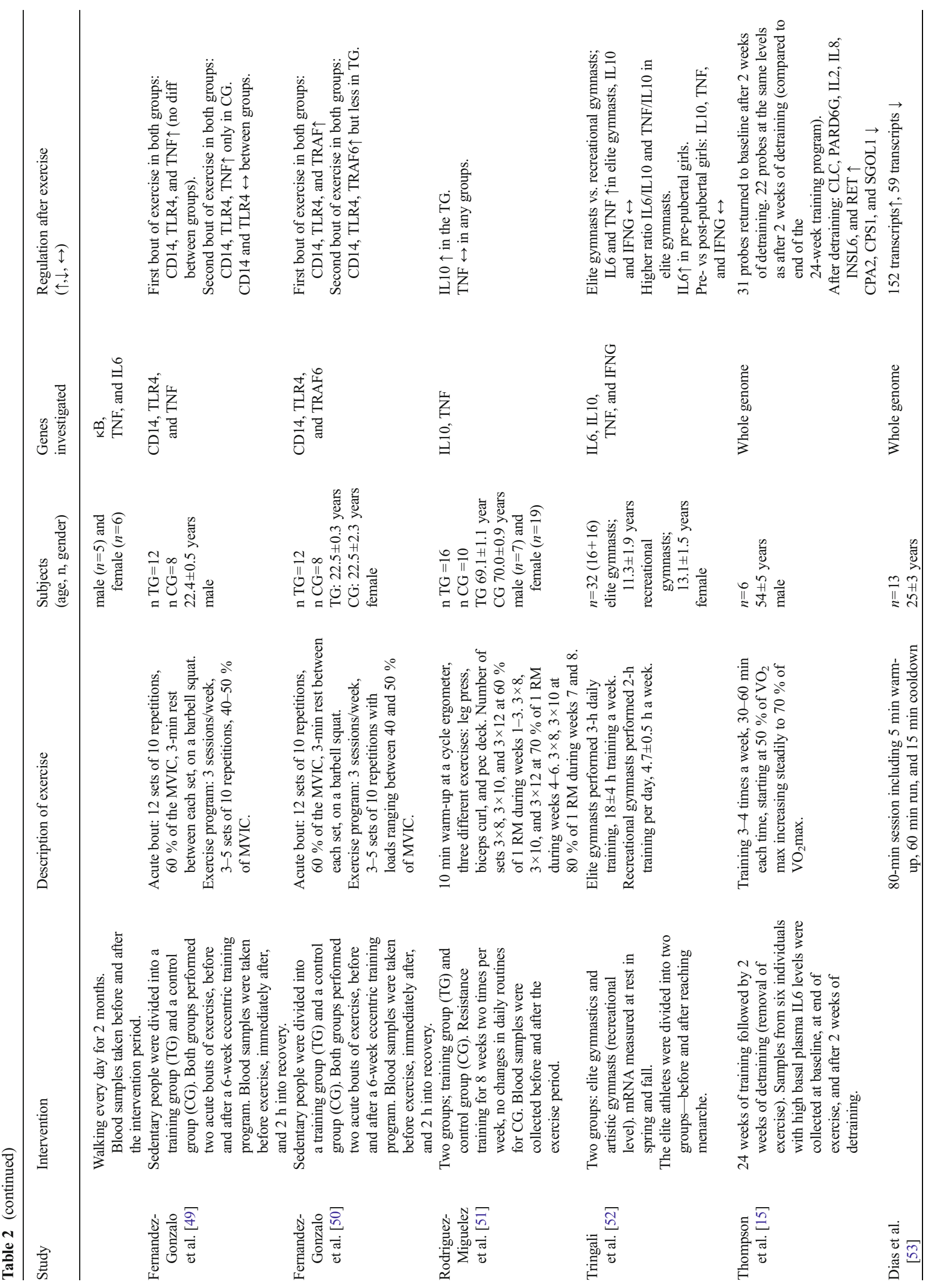




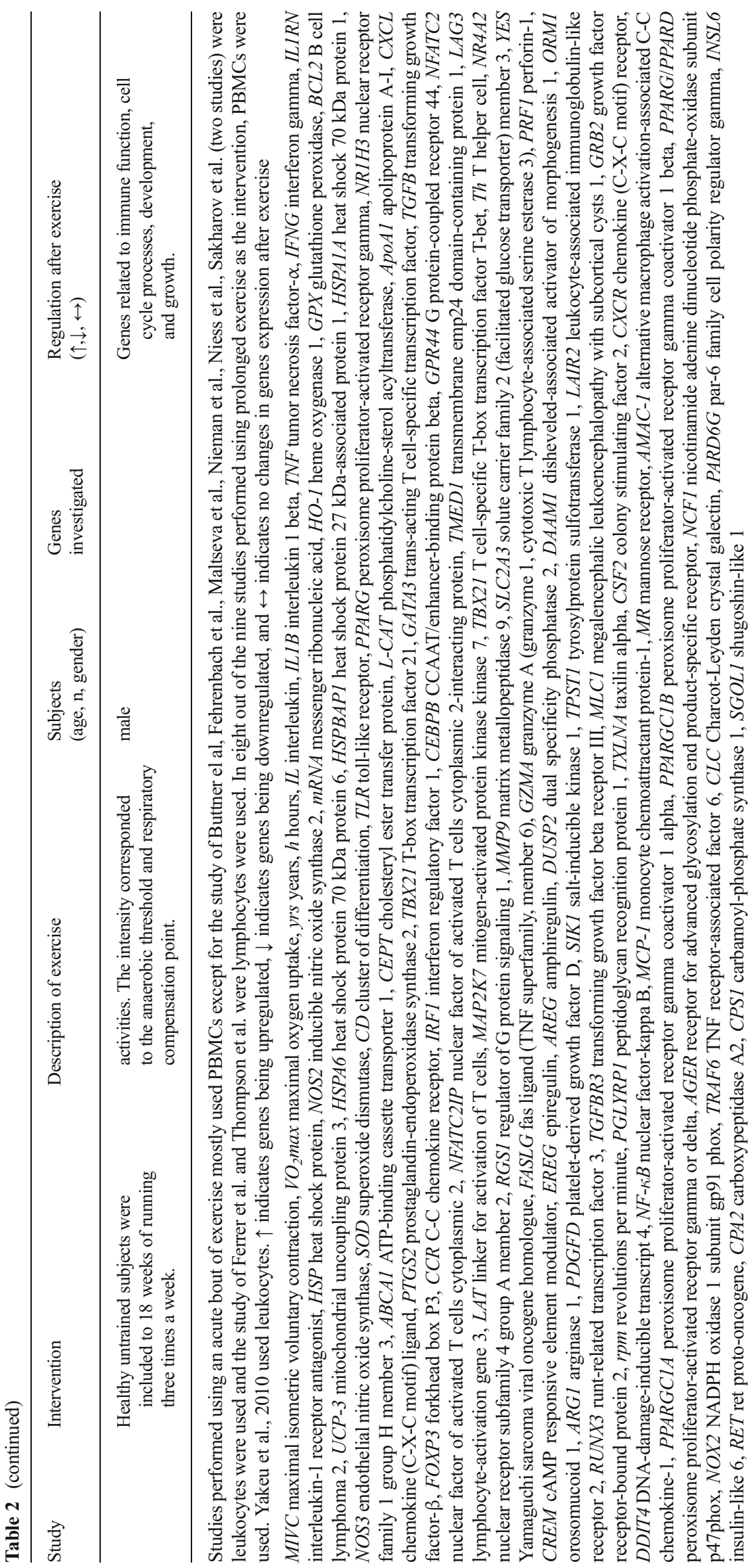


genes, related to the TGFB-signaling pathway, that were differently regulated in PBMCs after exercise [41]. Only runtrelated transcription factor 3 (RUNX3), transforming growth factor, beta receptor III (TGFBR3), megalencephalic leukoencephalopathy with subcortical cysts 1 (MLC1), and growth factor receptor-bound protein 2 (GRB2) were significantly altered.

Even though these studies are differently designed and the exercise programs are of different duration and intensities, exercise seems to have an influence on PBMCs. Genes regulated in these studies are associated with stress, inflammation, and tissue repair.

\section{Gene Expression Studies in Leukocytes-Endurance Training}

Heat shock proteins (HSP) have important functions as molecular chaperons and are produced in response to different stressful stimulus. It has also been shown that HSPs are able to function as powerful cytokines [57] by binding to TLR2 and TLR4 [51, 57].

Fehrenbach et al., Maltseva et al., and Sakharov et al. investigated how exercise might affect gene expression of HSPs after exercise. Fehrenbach et al. showed that mRNA expression of heat shock protein $70 \mathrm{kDa}$ binding protein (HSPBP1) increased in athletes compared to untrained subjects after exercise [58], while Maltseva et al. found that running altered the gene expression of peptidoglycan recognition protein (PGLYRP1) and heat shock protein $70 \mathrm{kDa}$ protein $1 \mathrm{~A}$ (HSPA1A), but not of HSPBP1 [42]. Sakharov et al. found an increased gene expression of HSPA1A after a treadmill test [29].

Subjects already participating in leisure activities performed a strenuous treadmill exercise, followed by a moderate treadmill exercise two weeks later in a study performed by Buttner et al. [39]. The mRNA levels of several genes were upregulated in both types of exercise, but there were differences depending on training intensity. Upregulated genes belonged to pathways associated with inflammation, stress signaling, electrolyte and substrate transport, extracellular matrix, and transcription factors.

Training intensity was also studied by Sakharov et al. who showed that 310 genes were upregulated in skiers after performing an exhausting treadmill test (RTE), while 69 genes were upregulated in the same subjects after performing a moderate treadmill test (MT) [43]. Sixty-four of the genes were identical in the RTE and MT, indicating a greater change in gene expression in response to a strenuous exercise.

These studies show that an acute bout of exercise alters gene expression in leukocytes and that exercise intensity might influence the level of inflammatory markers. The HSP may also play an important role in the acute response to exercise, possibly by inducing TLRs [51].

\section{Gene Expression Studies in Monocytes and Lymphocytes-Endurance Training}

Ferrer et al. wanted to assess the effects of swimming on the pro- and antioxidant system of lymphocytes [28]. They found an increase in gene expression of B cell CLL/lymphoma 2 (BCL2) and uncoupling protein (UCP)-3 after one hour swimming. No changes were seen in the expression of catalase, glutathione catalase (GPX), or peroxisome proliferator-activated receptor gamma, coactivator 1 alpha (PPARGC1A).

Heme oxygenase 1 (HO-1) is suggested to have both immune-protective and anti-inflammatory properties [27]. Thompson et al. performed a cross-over study were invited subjects ran for $75 \mathrm{~min}$, and the following test day were sitting calmly in the laboratory. The HO-1 mRNA expression level increased after exercise, while no changes were seen when resting in the laboratory [27]. The results from Thompsons' study [27] was confirmed by Sureda et al. who showed that HO-1 gene expression increased after moderate- and highintensity endurance training in soccer players [30].

Thomas et al. investigated if exercise was associated with an activation of peroxisome proliferator-activated receptor gamma (PPARG) signaling in monocytes in response to an acute bout of exercise [34]. The mRNA expression level of thrombospondin receptor (CD36), nuclear receptor subfamily 1 , group $\mathrm{H}$, member $3(\mathrm{NR} 1 \mathrm{H} 3)$ and ATP-binding cassette, subfamily A, member 1 (ABCA1) were upregulated while the expression levels of PPARG, PPARGC1A, cholesteryl ester transfer protein (CETP), and lecithin-cholesterol acyltransferase1 (L-CAT) were unchanged. These genes are key regulators of lipid and energy metabolism, which also are closely linked to inflammation. Radom-Aizik et al. found that the mRNA levels of TNF, TLR4, and CD36 were downregulated after an acute bout of exercise in monocytes [45] contradicting the results of Thomas et al. [34].

In the studies mentioned above, several genes known to be involved, or related to the immune system, have been investigated, both in males and females, young and elderly, and at different exercise intensities. All factors appear to have an effect on the outcome measured [59]. Few studies have been performed investigating the effects of gender and age. Some more studies have been performed studying differences in training intensities, making Sakharov et al. hypothesize that passing the anaerobic threshold is responsible for the differences seen in gene expression in white blood cells between low- and highintensity activities [43]. 


\section{Gene Expression Studies in PBMCs After Acute Resistance Exercise}

Only two studies have been looking at gene expression in response to an acute bout of resistance exercise, both using a microarray approach. Carlson et al. recruited healthy men to perform $30 \mathrm{~min}$ of resistance training [40]. Several genes were regulated in response to the exercise, and the greatest transcriptional changes were seen in genes related to immune response, cellular communication, and matrix remodeling. These results are largely supported by Storey et al. who found that 202 genes, primarily involved in cell-to-cell signaling, immune cell trafficking, organism survival, cell cycle, and cell death, were regulated after strength exercise [46].

The study of Carlson et al. [40] and Storey et al. [46] indicate that also strength exercise gives an acute inflammatory response. Given the differences in muscle work between endurance and strength exercise, one might expect a different inflammatory response, although some of the same pathways appear to be regulated as indicated by these two studies.

\section{Prolonged Exercise and Gene Expression Studies}

Table $2 b$ summarizes the effects of prolonged exercise on gene expression in white blood cells from nine studies, seven of which were performed using PBMCs and two using leukocytes. In studies using strength exercise as the intervention (four studies), mRNA expression level was measured before and after an acute bout of exercise, both at baseline and after the intervention period. In the remaining studies, gene expression was measured once before and once after the training period.

\section{Gene Expression Studies in PBMCs and Leukocytes-Endurance Exercise}

PBMC gene expression levels of IL6 and TNF in elite and recreational gymnasts were investigated by Tringali et al. [52]. The gene expression levels of IL6 and TNF were higher in elite gymnasts than in recreational gymnasts after half a year with exercise. Tringali et al. also showed that the IL6 gene expression level was higher in pre-pubertal girls than in girls having reached the menarche, and that the ratios of IL6/IL10 and TNF/IL10 were higher in elite gymnasts than in recreational gymnasts [52].

Eight weeks of a low-intensity training program elicited an increase in gene expression of PPARGC1A, peroxisome proliferator-activated receptor gamma, coactivator 1 beta (PPARGC1B), IL4, and CD 14 molecule (CD14) in leukocytes, while the mRNA expression levels of IL6, CXCL2,
TNF, and CCL2 were downregulated in a study performed by Yakeu et al. [48].

Dias et al. used a microarray approach when discovering that 211 gene transcripts related to immune function, cell cycle processes, development, and growth were regulated in PBMCs after 18 weeks of endurance training. One hundred and fifty-two gene transcripts were upregulated while 59 were downregulated [53].

Thompson et al. took another perspective when they examined the effect of an exercise intervention followed by two weeks without training [15]. Fifty-three gene transcripts, primarily involved in cell cycling, cell-mediated immune response, and cell-to-cell signaling and interactions, were differently regulated after the exercise period. After a period of detraining, the expression levels of 22 of the 53 genes were unaltered while the expression of 31 genes returned to baseline.

Even though the abovementioned studies are performed using different subjects (male and female, young and elderly), the results indicate that regular and moderate endurance exercise seems to lower some of the pro-inflammatory markers and/or promote an anti-inflammatory profile in the body.

\section{Gene Expression Studies in PBMCs-Resistance Exercise}

Jiménez-Jiménez et al. investigated the inflammatory response in PBMCs in elderly men before and after an eccentric training program [47]. Following both the first and the second bout of exercise, an increase in gene expression of NOS2 and PTGS2 were observed. IL6 mRNA increased after the first bout of exercise only. An attenuation of the acute inflammatory response after the training period for NOS2 and PTGS2 following the second exercise bout were observed.

Fernandez-Gonzalo et al. investigated how gene expression levels of CD14 and TLR4 were regulated in response to a bout of eccentric exercise performed before and after a training program in men [49] and women [50]. The mRNA expression levels of TNF and TNF receptor-associated factor 6 (TRAF6) were also studied in males and females, respectively. The acute bout increased the gene expression of CD14 and TLR4 in both male and female. The gene expression levels of TNF in men and TRAF6 in females were also increased after the first bout of exercise. The training period did not influence the acute response of CD14 or TLR4 mRNA gene expression, but the mRNA levels of TNF and TRAF6 were attenuated in males and females, respectively, after the second bout of exercise compared to the first bout in trained subjects.

When investigating the gene expression of IL10 and TNF after a resistance exercise program, Rodriguez-Miguelez et al. found an increase in the gene expression level of IL10 in the training group compared to the control group [51]. The 
authors concluded that resistance exercise may represent an effective tool to lower the pro-inflammatory status through an increased IL10/TNF-ratio.

Gano et al. found that the mRNA expression levels of advanced glycosylation end product-specific receptor (AGER), neutrophil cytosolic factor 1 (NCF1), and CCL2 were downregulated after an exercise period, improving the inflammatory/oxidative gene expression profile after a training period [16].

In all the studies using strength exercise, an acute bout of exercise has been included both at baseline and after exercise. With this design, the investigators will get valuable information about how one subject reacts to an acute bout of exercise while being trained compared to being untrained. This might be a valuable approach to study the molecular adaptions to exercise.

There are few studies performed investigating the effects of prolonged exercise on white blood cells and inflammation. The studies performed indicate that both resistance and endurance exercise promote an anti-inflammatory environment, or attenuate the acute response seen after a period of regular exercise.

\section{Discussion}

Epidemiological studies have shown that regular physical activity improves health. The molecular mechanisms behind the beneficial effects have however not been completely elucidated. A number of factors such as individual genetic variability, different exercise protocols, the heterogeneous nature of exercise itself, and other lifestyle factors influence the effects observed [5]. Furthermore, exercise exerts a number of effects on the body such as improving insulin sensitivity and lipid profile in addition to lowering blood pressure [5]. All these factors influence each other making it hard to understand the complex interactions.

Exercise causes damage to the muscle resulting in disarrangement in fiber structures, loss of fiber integrity, and leakage of muscle protein. Trying to restore homeostasis, several repair processes starts, involving inflammation, resolution, muscle repair, and finally regeneration. The balance between pro- and anti-inflammatory cytokines and other signaling molecules seems to be important for the outcome of the repair and regenerating process [60].

It has been recognized that the muscle is an endocrine organ being able to influence other organs such as the immune system [61]. The immune system is also directly involved in the cellular and molecular events in the muscle after exercise by recruitment of macrophages, neutrophils, and lymphocytes participating in the clearance of necrotic tissue and producing signaling molecules [60]. It is therefore plausible to believe that exercise may induce a response in PBMCs [24]. It is also possible that PBMCs are influenced by the exercise itself, since the immune system is directly involved in the repair processes of the muscle after exercise [36]. Studying how exercise affects PBMC gene expression to elucidate the molecular mechanisms of exercise is therefore highly relevant.

Exercise seems to induce responses both in the innate and in the adaptive immune system. It appears to influence both signaling molecules and transcription factors, as shown in many of the studies included in this review. The innate and the adaptive immune systems mutually affect each other, even though the first reaction most likely occurs in the innate immune system. The response to exercise seems to be closely regulated and both pro- and inflammatory cytokines and signaling molecules are released.

TLRs are important constituents of the innate immune system. They are located at the cell surface and may be stimulated by endogenous molecules that potentially arise during exercise, such as HSPs [57] and interleukins [56]. An activation of the TLR may further elicit an immune response resulting in an upregulation of pro-inflammatory cytokines and chemokines, involving the NF- $\mathrm{KB}$ and MAPK-pathways.

Nickel et al. [33], Ulven et al. [36], and Fernandez-Gonzalo et al. $[49,50]$ investigated the gene expression levels of TLRs after exercise. Their results are somewhat conflicting, but show that gene expression levels of TLRs are affected by exercise. The explanation for the different results might be due to the type of exercise performed, the training intensities, and the training status of the individuals - all shown to influence the inflammatory response [62].

$\mathrm{NF}-\mathrm{kB}$ is a transcription factor regulating a large number of genes, not only related to immune response, but also to cell survival, differentiation, and proliferation. NF-KB is expressed in all cells and is involved in both the innate and the adaptive immune system [63]. The pro-inflammatory effects of NF-KB are well known, but there are also evidence indicating an effect of NF- $\mathrm{KB}$ signaling in the resolution of an inflammatory response [64]. There is some evidence that NF- $\mathrm{kB}$ play an important role in the development of atherosclerosis [65].

NF- $\mathrm{kB}$ signaling pathways may be triggered by several stimuli such as NOS2 [56], HO-1 [30], TLRs [63], HSPs [57], TRAF [66], and cytokines such as IL1B and TNF [63], all genes investigated in articles included in this review [16, $24,26,27,29,30,47,50]$. Some of the results are conflicting, which may be due to the differences in training intensities.

NADPH oxidases play an important role in the innate immune system. NADPH oxidases are enzymes producing superoxide $\left(\mathrm{O}_{2}{ }^{-}\right)$, which again may produce reactive free radicals (ROS), thus being a possible contributor to atherosclerosis. It is hypothesized that the NCF1 play a role in TNF signaling and that impairing its expression may lower the oxidative and inflammatory status [67]. Gano et al. showed that the gene expression levels of NCF1 and NADPH oxidase 1, 
subunit gp91 phox (NOX2) were downregulated in PBMCs after two months of brisk walking [16], supporting this hypothesis.

Superoxide dismutases (SODs) are important molecules in the body's defense against $\mathrm{O}_{2}{ }^{-}$and free radicals [68]. SODs are able to catalyze the $\mathrm{O}_{2}{ }^{-}$radical into regular oxygen or hydrogen peroxide $\left(\mathrm{H}_{2} \mathrm{O}_{2}\right)$ and further to $\mathrm{H}_{2} \mathrm{O}$. Jenkins et al. investigated changes in mRNA levels of SODs in sedentary and endurance-trained athletes after a treadmill test [31], and found an increase in SOD1 and SOD2 gene expression in CD $34^{-}$cells after exercise in the sedentary group.

There are two main classes of lymphocytes that are important in the adaptive immune responses. B lymphocytes are primarily responsible for the antibody responses, while $\mathrm{T}$ lymphocytes are responsible for the cell-mediated immune responses [69].

T lymphocytes may be divided into subsets such as T helper 1 (Th1) cells or the humoral/antibody Thelper 2 (Th2) cells. Th1 cells primarily produce pro-inflammatory cytokines, while Th2 cells primarily produce anti-inflammatory cytokines [70,71]. These responses are well-documented, but they are not the only cytokine pattern possible [70, 72]. It seems like the Th1-Th2 decision is important for proper immune function [70] and that the NF-KB pathway is involved in the regulation of this differentiation $[20,63]$.

When comparing studies looking at the inflammatory response to exercise, it is important to compare subjects at the same physical level. Capomaccio et al. [73] and Fehrenbach et al. [24] found different expression levels of IL6 and heat shock protein $27 \mathrm{kDa}$-associated protein 1 (HSPBAP1) in highly trained athletes compared to lightly trained and untrained subjects at rest. Different baseline values may cause a different inflammatory response after exercising. Comparison among different cells types should also be done with caution as different cell types might respond differently to exercise [74].

There is also evidence indicating that the immune function and the response to exercise is altered with age $[5,75]$ and across puberty $[12,76]$. Elderly people also seem to have a higher basal level of inflammatory markers than younger people $[16,51,77]$.

\section{Concluding Remarks and Future Perspectives}

The effects of exercise on the body are multitudinous. Even though the study designs, the groups included, and the types of exercise used in the studies included in this review varies, it seems reasonable to conclude that exercise has an effect on cells of the immune system. Genes regulated after exercise are involved in inflammation, cellular communication, signal transduction, cellular protection, growth, and repair.
Overall, these studies show that an acute bout of exercise induces an immediate pro-inflammatory response, but that changes also occur in some of the anti-inflammatory markers. Prolonged and regular physical activity seems to promote an anti-inflammatory environment or attenuating the acute response to exercise, possibly reducing the risk of developing inflammatory-related diseases such as atherosclerosis $[14,78]$.

More research, preferably long-term standardized mechanistic studies, is needed to understand the impact of the observed change in gene expression, both in the muscles and in other organs, and to elucidate the complex interaction between white blood cells and muscle inflammatory response.

Financial Disclosure $\mathrm{KBH}$ and SMU have received research grant from Tine SA, Mills DA and Olympic Seafood.

\section{Compliance with Ethics Guidelines}

Conflict of Interest Gyrd Omholt Gjevestad is employed in TINE SA. Kirsten Holven reports grants from TINE BA, grants from Mills, grants from Olympic seafood, outside the submitted work. Stine Marie Ulven reports grants from TINE SA, grants from Mills, grants from Olympic seafood, outside the submitted work. This work was supported by grants from the Throne Holst Foundation for Nutrition Research; the University of Oslo, Oslo; and Akershus University College of Applied Sciences.

Human and Animal Rights and Informed Consent This article does not contain any studies with human or animal subjects performed by any of the authors.

Open Access This article is distributed under the terms of the Creative Commons Attribution 4.0 International License (http:// creativecommons.org/licenses/by/4.0/), which permits unrestricted use, distribution, and reproduction in any medium, provided you give appropriate credit to the original author(s) and the source, provide a link to the Creative Commons license, and indicate if changes were made.

\section{References}

1. Ross R. Atherosclerosis - an inflammatory disease. N Engl J Med. 1999;340(2):115-26.

2. Lee IM et al. Effect of physical inactivity on major noncommunicable diseases worldwide: an analysis of burden of disease and life expectancy. Lancet. 2012;380(9838):219-29.

3. Ahmed HM et al. Effects of physical activity on cardiovascular disease. Am J Cardiol. 2012;109(2):288-95.

4. Roberts CK, Barnard RJ. Effects of exercise and diet on chronic disease. J Appl Physiol (1985). 2005;98(1):3-30.

5. Woods JA, Vieira VJ, Keylock KT. Exercise, inflammation, and innate immunity. Neurol Clin. 2006;24(3):585-99.

6. Baynard $\mathrm{T}$ et al. Exercise training effects on inflammatory gene expression in white adipose tissue of young mice. Mediators Inflamm. 2012;767953. doi:10.1155/2012/767953.

7. Cruzat VF, Krause M, Newsholme P. Amino acid supplementation and impact on immune function in the context of exercise. J Int Soc Sports Nutr. 2014;11(1):61.

8. Peake J, Della Gatta P, Cameron-Smith D. Aging and its effects on inflammation in skeletal muscle at rest and following exercise- 
induced muscle injury. Am J Physiol Regul Integr Comp Physiol. 2010;298(6):R1485-95.

9. Bruunsgaard $\mathrm{H}$ et al. Exercise-induced increase in serum interleukin-6 in humans is related to muscle damage. J Physiol. 1997;499(Pt 3):833-41.

10. Karalaki $\mathrm{M}$ et al. Muscle regeneration: cellular and molecular events. In Vivo. 2009;23(5):779-96.

11. Smith LR, Meyer G, Lieber RL. Systems analysis of biological networks in skeletal muscle function. Wiley Interdiscip Rev Syst Biol Med. 2013;5(1):55-71.

12. Radom-Aizik $\mathrm{S}$ et al. Brief bout of exercise alters gene expression in peripheral blood mononuclear cells of early- and late-pubertal males. Pediatr Res. 2009;65(4):447-52.

13. Radom-Aizik $\mathrm{S}$ et al. A brief bout of exercise alters gene expression and distinct gene pathways in peripheral blood mononuclear cells of early- and late-pubertal females. J Appl Physiol (1985). 2009;107(1):168-75.

14. Libby P. Inflammatory mechanisms: the molecular basis of inflammation and disease. Nutr Rev. 2007;65(12 Pt 2):S140-6.

15. Thompson $\mathrm{D}$ et al. Time course of changes in inflammatory markers during a 6-mo exercise intervention in sedentary middleaged men: a randomized-controlled trial. J Appl Physiol (1985). 2010;108(4):769-79.

16. Gano LB et al. Increased proinflammatory and oxidant gene expression in circulating mononuclear cells in older adults: amelioration by habitual exercise. Physiol Genomics. 2011;43(14):895-902.

17. Pasterkamp G, Daemen M. Circulating cells: the biofactory for markers of atherosclerotic disease. Eur Heart J. 2008;29(22): 2701-2.

18. Visvikis-Siest $\mathrm{S}$ et al. Peripheral blood mononuclear cells (PBMCs): a possible model for studying cardiovascular biology systems. Clin Chem Lab Med. 2007;45(9):1154-68.

19. Hoefer IE et al. Circulating cells as predictors of secondary manifestations of cardiovascular disease: design of the CIRCULATING CELLS study. Clin Res Cardiol. 2013;102(11):847-56.

20. Hayden MS, West AP, Ghosh S. NF-kappaB and the immune response. Oncogene. 2006;25(51):6758-80.

21. Ullum $\mathrm{H}$ et al. Bicycle exercise enhances plasma IL6 but does not change IL-1 alpha, IL-1 beta, IL6, or TNF-alpha pre-mRNA in BMNC. J Appl Physiol (1985). 1994;77(1):93-7.

22. Natelson $\mathrm{BH}$ et al. Effect of acute exhausting exercise on cytokine gene expression in men. Int J Sports Med. 1996;17(4):299-302.

23. Ostrowski $\mathrm{K}$ et al. Evidence that interleukin- 6 is produced in human skeletal muscle during prolonged running. J Physiol. 1998;508(Pt 3):949-53.

24. Fehrenbach E et al. Transcriptional and translational regulation of heat shock proteins in leukocytes of endurance runners. J Appl Physiol. 2000;89(2):704-10.

25. Moldoveanu AI, Shephard RJ, Shek PN. Exercise elevates plasma levels but not gene expression of IL-1 beta, IL6, and TNF-alpha in blood mononuclear cells. J Appl Physiol (1985). 2000;89(4):1499_ 504.

26. Niess AM et al. Expression of the inducible nitric oxide synthase (iNOS) in human leukocytes: responses to running exercise. Med Sci Sports Exerc. 2000;32(7):1220-5.

27. Thompson D et al. Exercise-induced expression of heme oxygenase-1 in human lymphocytes. Free Radic Res. 2005;39(1): 63-9.

28. Ferrer MD et al. Antioxidant regulatory mechanisms in neutrophils and lymphocytes after intense exercise. J Sports Sci. 2009;27(1): 49-58.

29. Sakharov DA et al. Short-term highly intense physiological stress causes an increase in the expression of heat shock protein in human leukocytes. Bull Exp Biol Med. 2009;147(3):361-5.
30. Sureda A et al. Effects of exercise intensity on lymphocyte $\mathrm{H} 2 \mathrm{O} 2$ production and antioxidant defences in soccer players. Br J Sports Med. 2009;43(3):186-90.

31. Jenkins NT et al. Effects of acute and chronic endurance exercise on intracellular nitric oxide and superoxide in circulating CD34(+) and CD34(-) cells. J Appl Physiol (1985). 2011;111(3):929-37.

32. Li H, Howk C, Geib RW. A pilot study on the effect of acute Tai Chi practice on peripheral blood cytokine expression in healthy volunteers. Biomed Sci Instrum. 2012;48:254-9.

33. Nickel T et al. Modulation of dendritic cells and toll-like receptors by marathon running. Eur J Appl Physiol. 2012;112(5):1699-708.

34. Thomas AW et al. Exercise-associated generation of PPAR ligands activates PPAR signaling events and upregulates genes related to lipid metabolism. J Appl Physiol. 2012;112(5):806-15.

35. Bernecker $\mathrm{C}$ et al. Evidence for an exercise induced increase of TNF-alpha and IL6 in marathon runners. Scand J Med Sci Sports. 2013;23(2):207-14.

36. Ulven SM et al. An acute bout of exercise modulate the inflammatory response in peripheral blood mononuclear cells in healthy young men. Arch Physiol Biochem. 2015;27:1-9. doi:10.3109/ 13813455.2014.1003566.

37. Xiang L, Rehm KE, Marshall Jr GD. Effects of strenuous exercise on Th1/Th2 gene expression from human peripheral blood mononuclear cells of marathon participants. Mol Immunol. 2014;60(2): 129-34.

38. Connolly PH et al. Effects of exercise on gene expression in human peripheral blood mononuclear cells. J Appl Physiol (1985). 2004;97(4):1461-9.

39. Buttner $\mathrm{P}$ et al. Exercise affects the gene expression profiles of human white blood cells. J Appl Physiol (1985). 2007;102(1):2636.

40. Carlson LA et al. Changes in transcriptional output of human peripheral blood mononuclear cells following resistance exercise. Eur J Appl Physiol. 2011;111(12):2919-29.

41. Kimsa $\mathrm{M}$ et al. Expression pattern of the transforming growth factor beta signaling genes in human peripheral blood mononuclear cells after exercise-inflammatory aspects. Am J Hum Biol. 2012;24(6): 859-62.

42. Maltseva DV et al. Effect of exercise on the expression of HSPBP1, PGLYRP1, and HSPA1A genes in human leukocytes. Bull Exp Biol Med. 2012;153(6):866-8.

43. Sakharov DA et al. Passing the anaerobic threshold is associated with substantial changes in the gene expression profile in white blood cells. Eur J Appl Physiol. 2012;112(3):963-72.

44. Kimsa $\mathrm{MC}$ et al. Differential expression of inflammation-related genes after intense exercise. Prague Med Rep. 2014;115(1-2):24 32.

45. Radom-Aizik $\mathrm{S}$ et al. Impact of brief exercise on circulating monocyte gene and microRNA expression: implications for atherosclerotic vascular disease. Brain Behav Immun. 2014;39:121-9.

46. Storey AG, et al. Stress responses to short-term intensified and reduced training in competitive weightlifters. Scand J Med Sci Sports. 2015.

47. Jimenez-Jimenez R et al. Eccentric training impairs NF-kappaB activation and over-expression of inflammation-related genes induced by acute eccentric exercise in the elderly. Mech Ageing Dev. 2008;129(6):313-21.

48. Yakeu $\mathrm{G}$ et al. Low-intensity exercise enhances expression of markers of alternative activation in circulating leukocytes: roles of PPARgamma and Th2 cytokines. Atherosclerosis. 2010;212(2): 668-73.

49. Fernandez-Gonzalo R et al. Effects of eccentric exercise on toll-like receptor 4 signaling pathway in peripheral blood mononuclear cells. J Appl Physiol (1985). 2012;112(12):2011-8. 
50. Fernandez-Gonzalo R et al. TLR4-mediated blunting of inflammatory responses to eccentric exercise in young women. Mediators Inflamm. 2014;2014:479395.

51. Rodriguez-Miguelez $\mathrm{P}$ et al. Role of toll-like receptor 2 and 4 signaling pathways on the inflammatory response to resistance training in elderly subjects. Age (Dordr). 2014;36(6):9734.

52. Tringali $\mathrm{C}$ et al. Protective role of 17-beta-estradiol towards IL6 leukocyte expression induced by intense training in young female athletes. J Sports Sci. 2014;32(5):452-61.

53. Dias RG et al. PBMCs express a transcriptome signature predictor of oxygen uptake responsiveness to endurance exercise training in men. Physiol Genomics. 2015;47(2):13-23.

54. Lea T. Immunologiske metoder og teknikker. Fagbokforlaget Vigmostad \& Bjørke AS, 3rd edition, 2006.

55. Gay NJ et al. Assembly and localization of toll-like receptor signalling complexes. Nat Rev Immunol. 2014;14(8):546-58.

56. McCarthy CG et al. Toll-like receptors and damage-associated molecular patterns: novel links between inflammation and hypertension. Am J Physiol Heart Circ Physiol. 2014;306(2):H184-96.

57. Asea A et al. HSP70 stimulates cytokine production through a CD14-dependant pathway, demonstrating its dual role as a chaperone and cytokine. Nat Med. 2000;6(4):435-42.

58. Fehrenbach E et al. Changes of HSP72-expression in leukocytes are associated with adaptation to exercise under conditions of high environmental temperature. J Leukoc Biol. 2001;69(5):747-54.

59. Gabriel H, Kindermann W. The acute immune response to exercise: what does it mean? Int J Sports Med. 1997;18 Suppl 1:S28-45

60. Philippou A et al. Cytokines in muscle damage. Adv Clin Chem. 2012;58:49-87.

61. Pedersen BK. Muscle as a secretory organ. Compr Physiol. 2013;3(3):1337-62.

62. Fehrenbach E. Multifarious microarray-based gene expression patterns in response to exercise. J Appl Physiol (1985). 2007;102(1): $7-8$.

63. Uwe S. Anti-inflammatory interventions of NF-kB signaling: potential applications and risks. Biochem Pharmacol. 2008;75(8): 1567-79.
64. Perkins ND, Gilmore TD. Good cop, bad cop: the different faces of NF-kappaB. Cell Death Differ. 2006;13(5):759-72.

65. Baker RG, Hayden MS, Ghosh S. NF-kappaB, inflammation, and metabolic disease. Cell Metab. 2011;13(1):11-22.

66. Verstrepen $\mathrm{L}$ et al. TLR4, IL-1R and TNF-R signaling to NFkappaB: variations on a common theme. Cell Mol Life Sci. 2008;65(19):2964-78.

67. Petry A, Weitnauer M, Gorlach A. Receptor activation of NADPH oxidases. Antioxid Redox Signal. 2010;13(4):467-87.

68. Fukai T, Ushio-Fukai M. Superoxide dismutases: role in redox signaling, vascular function, and diseases. Antioxid Redox Signal. 2011;15(6):1583-606.

69. Alberts B, Johnson A, Lewis J, et al. Molecular biology of the cell. 4th ed. New York: Garland Science; 2002.

70. Mosmann TR, Sad S. The expanding universe of T-cell subsets: Th1, Th2 and more. Immunol Today. 1996;17(3):138-46.

71. Matzinger P, Kamala T. Tissue-based class control: the other side of tolerance. Nat Rev Immunol. 2011;11(3):221-30.

72. Gleeson M. Immune function in sport and exercise. J Appl Physiol (1985). 2007;103(2):693-9.

73. Capomaccio $\mathrm{S}$ et al. Athletic humans and horses: comparative analysis of interleukin-6 (IL6) and IL6 receptor (IL6R) expression in peripheral blood mononuclear cells in trained and untrained subjects at rest. BMC Physiol. 2011;11:3.

74. Radom-Aizik $\mathrm{S}$ et al. Effects of 30 min of aerobic exercise on gene expression in human neutrophils. J Appl Physiol (1985). 2008;104(1):236-43.

75. Peake J, Nosaka K, Suzuki K. Characterization of inflammatory responses to eccentric exercise in humans. Exerc Immunol Rev. 2005;11:64-85.

76. Lamason $\mathrm{R}$ et al. Sexual dimorphism in immune response genes as a function of puberty. BMC Immunol. 2006;7:2.

77. Bruunsgaard $\mathrm{H}$. Effects of tumor necrosis factor-alpha and interleukin-6 in elderly populations. Eur Cytokine Netw. 2002;13(4):389-91.

78. Gleeson $\mathrm{M}$ et al. The anti-inflammatory effects of exercise: mechanisms and implications for the prevention and treatment of disease. Nat Rev Immunol. 2011;11(9):607-15. 